\title{
Indoor SLAM using a Range-Augmented Omnidirectional Vision
}

\author{
B. Bacca Cortes \\ University of Girona \\ Universidad del Valle \\ Girona, Building PIV, 17071 \\ bladimirdeia.udg. edu \\ evbaccadunivalle.edu.co
}

\author{
X. Cufí Solé \\ University of Girona \\ Girona, Building PIV, 17071 \\ xcuf@eia.udg.edu
}

\author{
J. Salví \\ University of Girona \\ Girona, Building PIV, 17071 \\ qsalvideia.udg.edu
}

\begin{abstract}
A promising combination of sensors as a laser range finder and an omnidirectional camera can be used to extract rich $3 D$ information from indoor environments for robot mapping and localization. This paper presents an implementation of FastSLAM using $3 D$ vertical edges retrieved from a range-augmented omnidirectional vision sensor. Our sensor model in conjunction with the FastSLAM algorithm solves the indoor Simultaneous Localization and Mapping problem. The real world experiment to validate our approach used a Pioneer-3DX mobile robot equipped with a URG-04LX laser range finder and an omnidirectional camera.
\end{abstract}

\section{Introduction}

Reliable self-localization and map building plays an important role for autonomous navigation in mobile robotics. Indoor environments are the scenery where service robots are useful for surveillance, inspection, delivery and cleaning tasks. Then, the perception system of a mobile robot must provide accurate information of the robot environment, taking advantage of its surroundings in order to reconstruct a consistent representation of the environment. In many applications this environment representation is not known and the mobile robot has to locate itself at the same time using a set of sequential observations. This problem is called Simultaneous Localization and Mapping (SLAM), which has been studied throughout many years and excellent surveys have written [1-2].

The most relevant solutions to SLAM are focused on the feature-based approach, where feature descriptors are extracted from laser scans or images to solve the problem of matching observations to landmarks. Other alternative approaches are: in [3] raw laser range data is used to extract saliency using the Iterative Closest Point (ICP) algorithm [4]; [5] computes the camera pose and the scene structure considering illumination changes using the image intensities as saliency.

In recent years, appearance-based mapping and localization has gained special attention since these methods use a richer description of the environment giving more cues to improve robot mapping and localization [67]. These approaches present a probabilistic framework to build appearance-based topological maps. Appearancebased methods exploit the environment structure as vertical and horizontal lines (laser scans or images), doors, planes, etc. to obtain a better representation of the robot surroundings. In this sense, [8] and [9] proposed solutions using only omnidirectional images and bearing information of vertical lines to solve SLAM, [10] described a system based on a laser range finder (LRF) and an omnidirectional camera to obtain a map of the environment using scan matching and vertical lines.

Another option to obtain better environment representations concerns sensor fusion. Common available laser range finders work in a plane parallel to the ground, and then it limits the environment representation to $2 \mathrm{D}$. Combining vision sensors with laser range finders increase the perceptual information, but monocular or stereo cameras have limited field of view affecting their perception due to occlusions and feature lifetime observation. Omnidirectional cameras have received special attention recently due to its long-term landmark tracking, its wide field of view, its robustness to occlusions, and its ability to be fused with range data. Therefore, combining an omnidirectional camera with a LRF has many advantages: all the laser trace can be used to extract environment features on the image plane, depth information can be embedded into the omnidirectional image, 3D feature information can be recovered, and once the calibration between these sensors is performed for first time it can be used in real time. In [11] a sensor fusion between an omnidirectional camera and a LRF is presented, where the laser trace projected onto the omnidirectional image is used to extract salient features on the image plane.

In this paper we present an implementation of the FastSLAM algorithm using a sensor model based on the extrinsic calibration between a LRF and an omnidirectional camera [12]. The main goal of our approach is to extract the 3D position of vertical lines in indoor environments and use them to solve the SLAM problem, instead of using the bearing information only as 
is done in [8] and [9]. Our approach uses a modified version of the joint compatibility branch and bound test (JCBB) [13] to solve the data association problem. The data association is performed using the features 3D position, instead of using local 1D image intensity signals as presented in [11]. The experimental validation was performed using a dataset collected in the indoors of the University of Girona.

This paper is organized as follows: Section 2 describes the SLAM algorithm we implemented. Section 3 concerns the description of the sensor, feature extraction and data association. Section 4 describes the scenario and the experimental results we obtained that validate our approach. The paper ends with conclusions presented in Section 5.

\section{SLAM algorithm}

One successful solution to SLAM is particle filters. Mapping and localization involves non-linear functions and unknown error sources, the latter cannot be modeled using an exact mathematical representation. In this context, particle filters can handle this kind of problems sampling from an estimate probabilistic distribution and improving it recursively. In this paper we used the FastSLAM 1.0 [14-15] algorithm, which uses particle filtering and we have adapted it to support our measurement model.

In our implementation the system state is composed of the robot position $\left(X=\left[x_{R}, y_{R}, \theta_{R}\right]\right)$ and the vertical lines position $(M)$, the observations are the range, azimuth and elevation of the vertical lines $(Z)$ and the control data $(U)$ is obtained from scan matching [4]. As conditionally independence can be assumed given the robot poses, the posterior can be factored as follows:

$p\left(x_{t}, M \mid z_{1: t}, u_{1: t}\right)=p\left(x_{t} \mid z_{1: t}, u_{1: t}\right) \prod_{n=1}^{N} p\left(m_{n} \mid x_{t}, z_{1: t}\right)$ (1)

Where, $t$ is the current time step and $N$ is the current number of features. In FastSLAM each particle is denoted by:

$y_{t}^{k}=\left\langle x_{t}^{k}, \mu_{1, t}^{k}, \Sigma_{1, t}^{k}, \ldots, \mu_{N, t}^{k}, \Sigma_{N, t}^{k}\right\rangle$

Where, $k$ is the particle index, $x_{t}^{k}$ is the path estimate of the robot, and $\mu_{n, t}^{k}$ and $\Sigma_{n, t}^{k}$ are the mean and variance of the Gaussian representing the $n$ - $t h$ feature location of the $k$-th particle which are estimated using independent Kalman filters. The filtering process overview is explained as follows:

Getting measures - Vertical lines position with respect to the LRF are gathered: $z_{n, t}=\left[\rho_{n, t} \phi_{n, t} \psi_{n, t}\right]^{T}$, where $\rho_{n, t}, \phi$ ${ }_{n, t}$ and $\psi_{n, t}$ are the range, the azimuth and elevation of the $n$-th vertical line at time $t$.
Sampling new poses - A new pose $x_{t}$ is sampled using scan-matching for each particle in $y_{t-1}$. This is done drawing a sample according to the motion posterior:

$x_{t}^{k} \sim p\left(x_{t}^{k} \mid x_{t-1}^{k}, u_{t}\right)$

Where, $x_{t-1}^{k}$ is the posterior estimate for the robot location at time $t-l$ in the $k$-th particle, and $u_{t}$ is the command motion obtained from scan-matching.

Data association - Given the current set of features for the $k$-th particle, the current set of observations $z_{t}$ and the current predicted pose $x_{t}^{k}$ from Equation 3, we use the JCBB test [13] in order to find the observed features $H_{t}$.

Update observed features - For each observed feature in each $k$-th particle, the standard EKF expressions were applied to obtain the mean $\left(\mu_{H_{t}, t}^{k}\right)$ and covariance $\left(\Sigma_{H_{t}, t}^{k}\right)$ of these features:

$K_{t}^{k}=\Sigma_{H_{t}, t-1}^{k} \cdot J_{t}^{k \cdot T}\left(J_{t}^{k} \cdot \Sigma_{H_{t}, t-1}^{k} \cdot J_{t}^{k \cdot T}+Q_{t}\right)^{-1}$

$\mu_{H_{t}, t}^{k}=\mu_{H_{t}, t-1}^{k}+K_{t}^{k}\left(z_{t}-\widehat{z_{t}^{k}}\right)$

$\Sigma_{H_{t}, t}^{k}=\left(I-K_{t}^{k} J_{t}^{k}\right) \cdot \Sigma_{H_{t}, t-1}^{k}$

Where, $J_{t}^{k}$ is the Jacobian of the measurement model with respect to the feature coordinates (see Section 3) and $Q_{t}$ is the sensor uncertainty.

Re-sampling - We use the FastSLAM low variance resampling method, where the importance factor of each particle depends on their measurement probability.

The main goal of this paper is propose an original measurement model, which is used in steps 1,3 and 4 of the FastSLAM algorithm described above. The measurement model proposed in this paper is explained in the next section.

\section{Sensor model}

A central catadioptric camera consists of a perspective or orthographic camera, and a mirror. The latter can be conic, hyperbolic, or parabolic. Projective models for these cameras have been developed by [16] and [17]. In the remainder of this paper we adopt the model described in [17], which is related to the toolbox described in [18], and use it to calibrate our central catadioptric camera.

The URG-04LX 2D LRF used in this work was previously calibrated in order to decrease the range error following the procedure described in [10]. The raw 2D laser scan data is previously processed using a median filter. Our approach was tested using a dataset with the robot in motion, for this reason a motion correction is performed in the 2D laser scan.

The observations we used to feed the FastSLAM algorithm are the vertical edges with respect to the LRF. These observations are the range, azimuth and elevation angles, which are obtained thanks to the extrinsic 
calibration between the omnidirectional camera and the LRF.

\subsection{Omnidirectional camera / laser range finder calibration}

Figure 1 depicts the extrinsic calibration between the omnidirectional camera and the LRF. There are three coordinate systems, those of the laser, the calibration pattern and the camera. The problem focuses on finding $R$ and $T$ so that laser points $P_{L}$ can be represented in the camera coordinate system, and then projected onto the omnidirectional image.

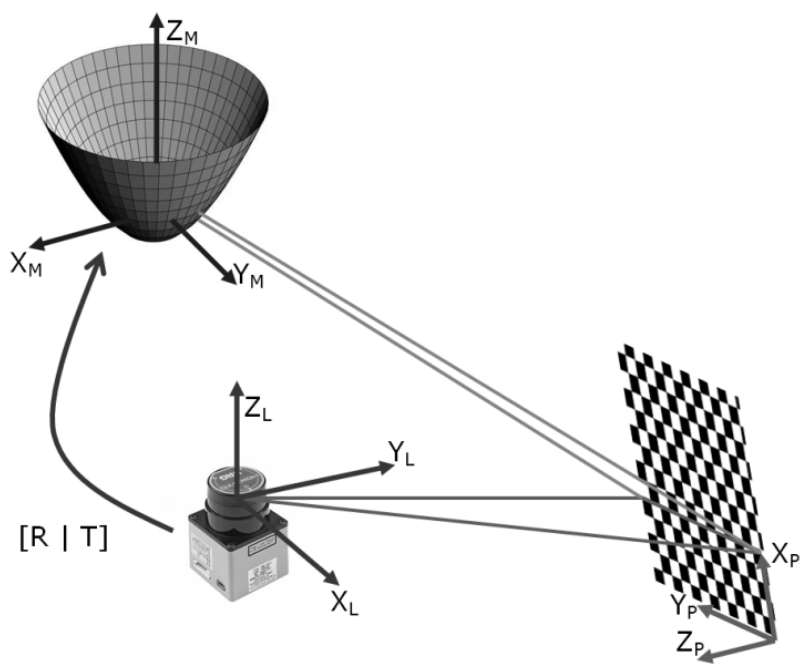

Figure 1. Omnidirectional camera and LRF extrinsic calibration.

Using the simultaneous parameter estimation proposed in [10] the $R$ and $T$ matrices were found obtaining the following results: $T=\left[\begin{array}{lll}0.0283 & -0.1984 & 0.55\end{array}\right]^{T}$ in meters and $-1.1075^{\circ},-3.4829^{\circ}$ and $-94.3732^{\circ}$ for the Roll, Pitch and Yaw angles.

\subsection{Vertical line detection}

For line detection we used a modified version of [19]. For self-containment, here follows just the main idea. This algorithm starts detecting edges in the image and building chains of connected edge pixels. Then these chains are projected on the sphere, where the great circle constraint is verified to be considered as a line. Otherwise, it is iteratively cut into 2 sub-chains until a sub-chain is considered a line or its length is too small. Then, a merging step is applied because a line might be decomposed into more than a single chain.

The approach presented in [19] detects any line; hence we modified the pipeline process in order to detect only the vertical lines. Besides, we included an additional constraint given by the cross-product between the sphere projection of the line and the normal ground plane. In addition, to improve the computing time the edge and chain detection were implemented in MEX files; and using the camera calibration a look-up-table (LUT) was computed to accelerate the image point projection on the sphere.

\subsection{Vertical line position}

Figure 2 shows how the vertical line position can be computed. In this figure two typical vertical lines are presented. The line $L_{1}$ crosses the LRF trace (dotted line) and the line $L_{2}$ does not. $A_{L 1}$ and $C_{L 1}$ are the initial and ending points of $L_{1} . B_{L l}$ is the LRF point associated to $L_{1}$. In order to describe the vertical line position method, we need to define the following angles: $\theta_{L 1}=\angle z O B_{L 1}, \theta_{A 1}=$ $\angle z O A_{L 1}$ and $\theta_{C l}=\angle z O C_{L 1}$.

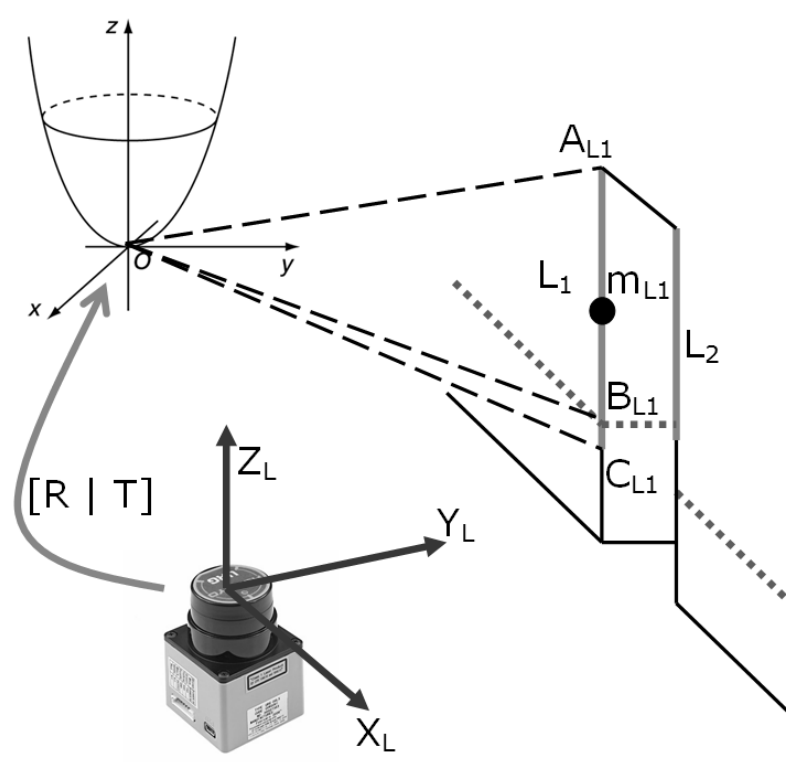

Figure 2. Vertical line position measurement.

The vertical lines detected are spread in the $360^{\circ}$ field of view of the omnidirectional image. However, the LRF has a limited field of view of just $240^{\circ}$. Then those vertical lines on the image out of the field of view of the LRF are filter out. Next, using the range uncertainty of the LRF $\left(\sigma_{\rho}\right.$ $=0.04)[20]$ each vertical line is associated to the nearest LRF point projected onto the omnidirectional image ( $B_{L 1}$ in Figure 2). Once the corresponding points $A_{L 1}, B_{L 1}$ and $C_{L 1}$ are found on the image plane, they are projected to the sphere and the angles $\theta_{L 1}, \theta_{A 1}$ and $\theta_{C l}$ can be computed. Then, scene points $A_{L 1}$ and $C_{L I}$ can be expressed as follows:

$A_{L 1}=\left[\begin{array}{l}x_{A} \\ y_{A} \\ z_{A}\end{array}\right]=\left[\begin{array}{c}\rho_{B} \cos \left(\theta_{L 1}\right) \\ \rho_{B} \sin \left(\theta_{L 1}\right) \\ T_{3}-\frac{\rho_{B}}{\tan \left(\theta_{A 1}\right)}\end{array}\right]$ 
$C_{L 1}=\left[\begin{array}{l}x_{C} \\ y_{C} \\ z_{C}\end{array}\right]=\left[\begin{array}{c}\rho_{B} \cos \left(\theta_{L 1}\right) \\ \rho_{B} \sin \left(\theta_{L 1}\right) \\ T_{3}-\frac{\rho_{B}}{\tan \left(\theta_{C 1}\right)}\end{array}\right]$

Where, $\rho_{B}$ is the distance to the vertical line extracted from the corresponding LRF point and $T_{3}$ is the third element of the translation vector between the omnidirectional camera and the LRF. Using these scene points the observation model can be computed as:

$Z_{n}=\left[\begin{array}{c}r_{n} \\ \phi_{n} \\ \psi_{n}\end{array}\right]=\left[\begin{array}{c}\sqrt{\left(m_{x}-x_{R}\right)^{2}+\left(m_{y}-y_{R}\right)^{2}+m_{z}^{2}} \\ \operatorname{atan} 2\left(\frac{m_{y}-y_{R}}{m_{x}-x_{R}}\right)-\theta_{R} \\ \cos ^{-1}\left(\frac{m_{z}}{r_{n}}\right)\end{array}\right]$

Where, $r_{n}, \phi_{n}$ and $\psi_{n}$ are the observed range, azimuth and elevation of the middle point $m_{L 1}=\left[m_{x}, m_{y}, m_{z}\right]$ of line $L_{1}$ given the current robot position $X_{t}=\left[x_{R}, y_{R}, \theta_{R}\right]$. To update the observation at step 4 of Section 2, EKF requires a linearized measurement model with additive Gaussian noise $\left(Q_{t}\right.$ in Equation 4). The Taylor approximation of this measurement model is:

$z_{t}^{k}\left(\mathrm{X}_{\mathrm{t}}^{\mathrm{k}}, m_{H_{t}}\right) \approx z_{t}^{k}\left(\mathrm{X}_{\mathrm{t}}^{\mathrm{k}}, \overline{m_{H_{t}, t-1}}\right)+J_{t}^{k}\left(m_{H_{t}}-\overline{m_{H_{t}, t-1}}\right)$

$J_{t}^{k}=\left[\begin{array}{ccc}\frac{d x}{r_{n}} & \frac{d y}{r_{n}} & \frac{d z}{r_{n}} \\ \frac{-d y}{d} & \frac{d x}{d} & 0 \\ \frac{d x}{d . f} & \frac{d y}{d . f} & \frac{-1}{\sqrt{d-d z^{2}}}\end{array}\right]$

Where, $J_{t}^{k}$ is the Jacobian with respect to the feature coordinates, $\mathrm{X}_{\mathrm{t}}^{\mathrm{k}}$ is the robot state of the $k$-th particle, $\overline{m_{H_{t}, t-1}}$ is the feature mean location in the previous time step, $d x=m_{x}-x_{R}, d y=m_{y}-y_{R}, d z=m_{z}, d=\left(m_{x}-\right.$ $\left.x_{R}\right)^{2}+\left(m_{y}-y_{R}\right)^{2}+m_{z}{ }^{2}$ and $f=\sqrt{\left(d / d z^{2}\right)-1}$. In our implementation of the FastSLAM algorithm we also update the robot position. Proceeding in the same way as before, the Taylor approximation is:

$$
\begin{aligned}
& z_{t}^{k}\left(\mathrm{X}_{\mathrm{t}}^{\mathrm{k}}, m_{H_{t}}\right) \approx z_{t}^{k}\left(\overline{\mathrm{X}_{\mathrm{t}}^{\mathrm{k}}}, m_{H_{t}}\right)+F_{t}^{k}\left(\mathrm{X}_{\mathrm{t}}^{\mathrm{k}}-\overline{\mathrm{X}_{\mathrm{t}}^{\mathrm{k}}}\right) \\
& F_{t}^{k}=\left[\begin{array}{ccc}
\frac{-d x}{r_{n}} & \frac{-d y}{r_{n}} & 0 \\
\frac{d y}{d} & \frac{-d x}{d} & -1 \\
\frac{-d x}{d . f} & \frac{-d y}{d . f} & 0
\end{array}\right]
\end{aligned}
$$

Where, $F_{t}^{k}$ is the Jacobian with respect to the robot state $\left(\mathrm{X}_{\mathrm{t}}^{\mathrm{k}}\right), \overline{\mathrm{X}_{\mathrm{t}}^{\mathrm{k}}}$ is the predicted robot state, and $d x, d y, d$ and $f$ have the same meaning as in Equation 11.

\subsection{Data association}

In our implementation we considered JCBB to solve the data association problem [13], such that the correlations between innovations are explicitly taken into account to determine the joint compatibility of a set of pairings. Each particle in the particle filter holds the robot path and the estimated positions of the vertical lines. A key advantage of using a particle filter is that each particle can perform its own data association decisions based on its robot path and landmark position estimates.

The measurement model proposed in this paper is focused on finding the range, azimuth and elevation of the vertical line middle point. However, this measure and the innovation gate distance metric used in JCBB do not exploit other landmark attributes, which may be useful for disambiguation. For this reason, additionally we extract the direction of the vertical line which includes: the initial point, the ending point and its length. Using Equations 7 and 8 , we can define the line direction $u_{L 1}$ of the vertical line $L_{l}$ :

$L_{1}=\left[\begin{array}{l}x_{A}+u_{L 1, x} l \\ y_{A}+u_{L 1, y} l \\ z_{A}+u_{L 1, z} l\end{array}\right]$

$u_{L 1}=\frac{C_{L 1}-A_{L 1}}{\left\|C_{L 1}-A_{L 1}\right\|}$

Where, $l$ varies from 0 to the vertical line length, which is defined by the norm of the vector $\overrightarrow{A_{L 1}-C_{L 1}}$. Once the feature correspondences are given by JCBB, we search those ambiguous correspondences and we filtered them out using the following criteria:

newH $H_{t}=\min _{c_{k}} W\left(c_{k}\right)$
$W\left(c_{k}\right)=\operatorname{JCdist}\left(c_{k}\right)\left|\frac{L\left(c_{k}\right)_{M A P}-L\left(c_{k}\right)_{H_{t}}}{L\left(c_{k}\right)_{M A P}}\right|$

Where, $c_{k}$ is the list of potential ambiguous correspondences, $\operatorname{JCdist}\left(c_{k}\right)$ is the joint compatibility associated to the map feature, $L\left(c_{k}\right)_{M A P}$ is the vertical line length stored in the map and $L\left(c_{k}\right)_{H t}$ is the observed vertical line length. In this way not only the correlations between innovations are taken into account, but also other landmark attributes as its length is considered.

\section{Real experiments}

We tested our approach on a Pioneer 3DX mobile robot equipped with an onboard computer at $1.5 \mathrm{GHz}$, an omnidirectional vision setup composed of a RemoteReality parabolic mirror with a diameter of $74 \mathrm{~mm}$, a UI-2230SE-C camera with a resolution of $1024 \times 768$ pixels, and a URG-04LX laser range finder (Figure 3).

We collected our data set at the PIV building of the University of Girona which has three floors. These data 
sets include the LRF readings, the corresponding omnidirectional images and the robot odometry. Figure 4 shows two columns of omnidirectional images, each column corresponds to the first and third floor of the PIV building, respectively. These images show the environmental conditions in which the data sets were collected. It can be observed that there are illumination changes and occlusions caused by pedestrians assuring in this way a real-world experiment. The data set corresponding to the first floor has a path length of $\sim 80 \mathrm{~m}$ and that on the third floor a length of $\sim 55 \mathrm{~m}$.

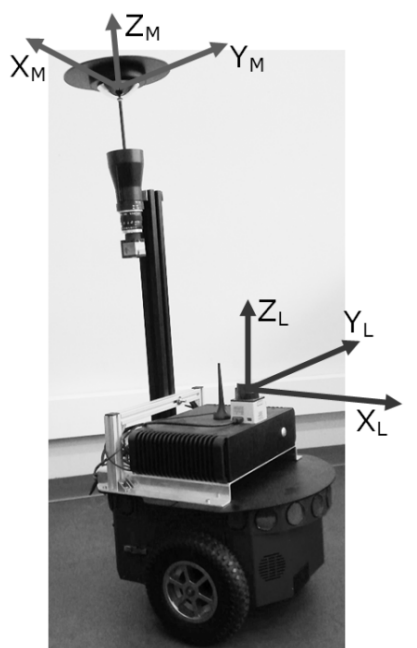

Figure 3. Robot platform and experimental setup.

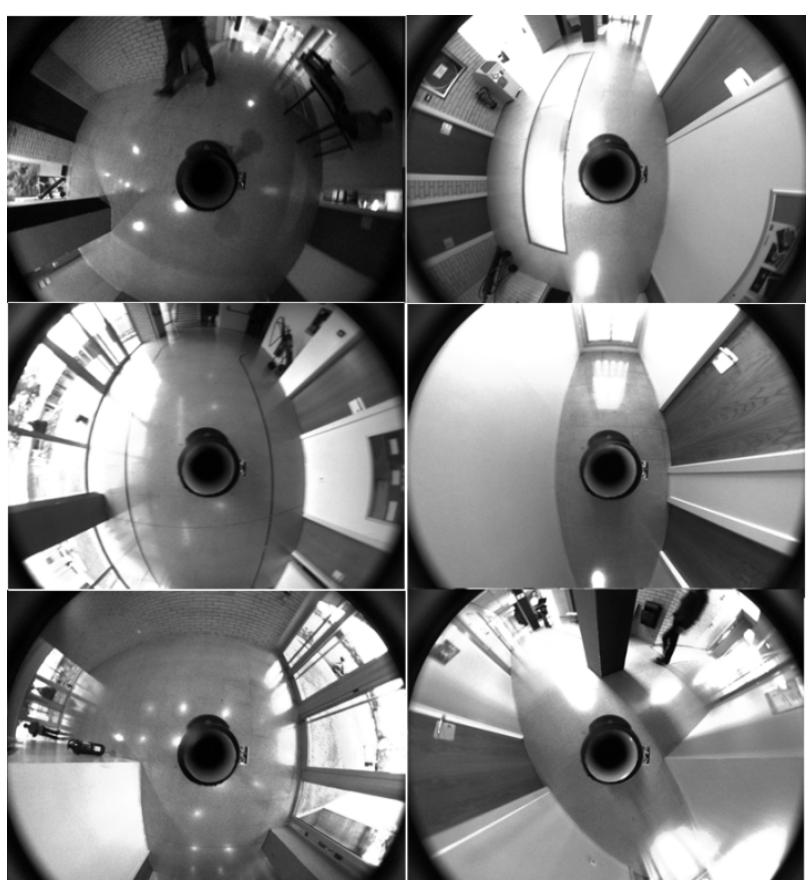

Figure 4. Omnidirectional images samples taken from the collected data set. Each column corresponds to the first and third floor of the PIV building, respectively.
Figure 5 shows two maps, the first one (top) was built using scan matching and the second one (bottom) depicts our estimated map, both on the CAD map of the first floor of the PIV building. Our estimated map shows the robot path and the landmarks estimated positions of the best particle. Comparing these both maps is clear that our approach obtains a better match with respect to the CAD drawing. However, a drift error due a rotation of $180^{\circ}$ is present on the top-right part of our estimated map. The dataset was taken in presence of pedestrians, who are shown by the arrows in both maps. Despite of this fact, our approach obtains a coherent representation of the environment.

Taking into account the first column of Figure 4, it is worth to note that the omnidirectional images correspond to the first loop at the left of Figure 5, the first corridor above the office No. 005 and the back exit of PIV building below the office No. 015 .

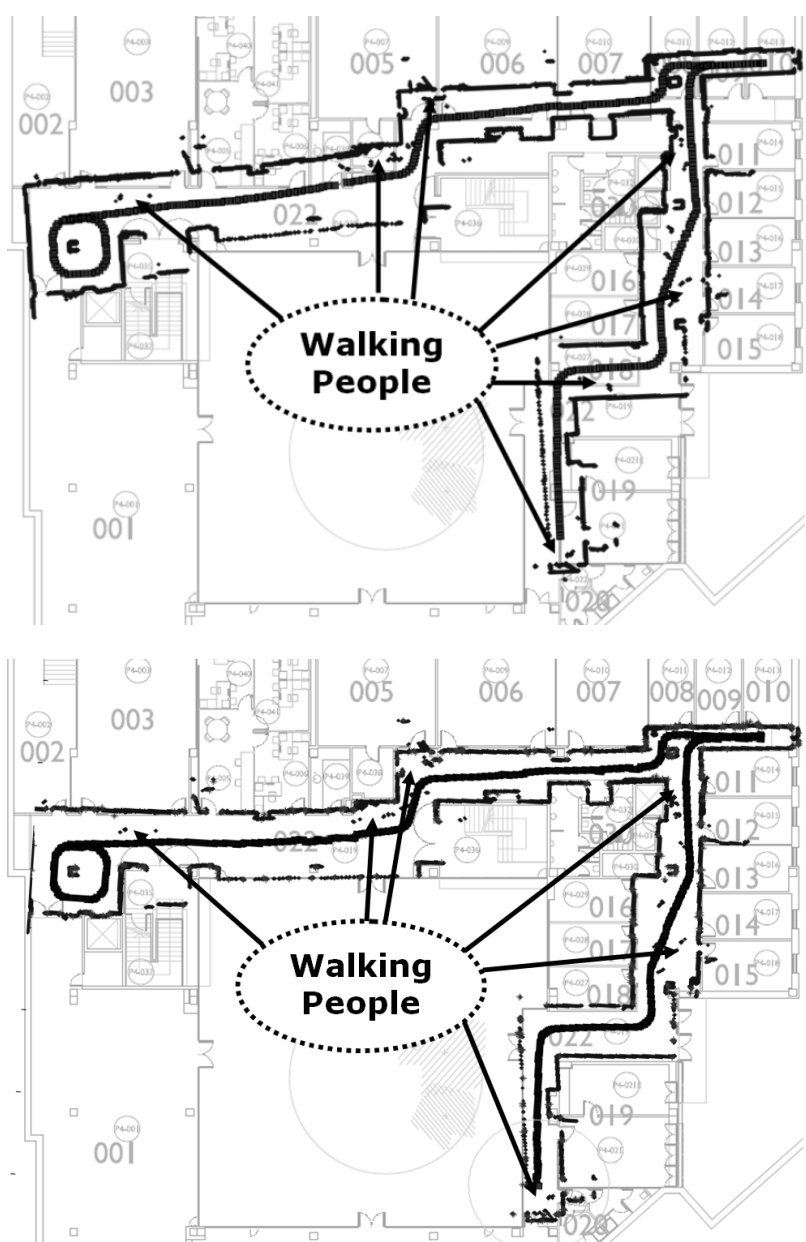

Figure 5. First experiment: SLAM at the first floor of PIV building. Top: scan matching map. Bottom: our estimated map.

Figure 6 shows a zoomed region of Figure 5. This figure shows the consistency between the laser scan 
matching and the corresponding vertical lines in the scene. However, in some cases the windows reflections on the floor caused false vertical lines which have to be filter out. An example of this situation can be observed in the first and second image, second column of Figure 4.

Using our approach, one omnidirectional image and the corresponding LRF trace are only needed in order to obtain a local 3D reconstruction of the scene. This has many advantages as: it avoids dealing with the scale factor problem when only a vision sensor is used, it gives metric information in real time and it solves disambiguation caused by dynamic environments.

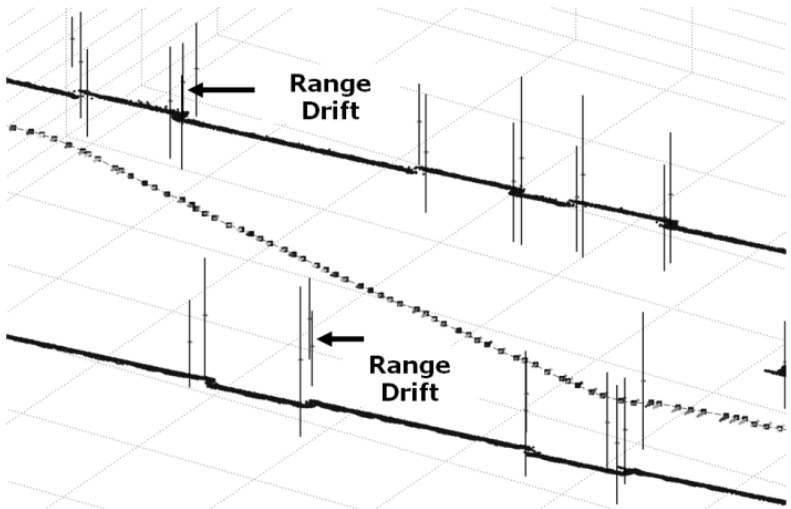

Figure 6. Details of Figure 5 with 3D vertical lines.
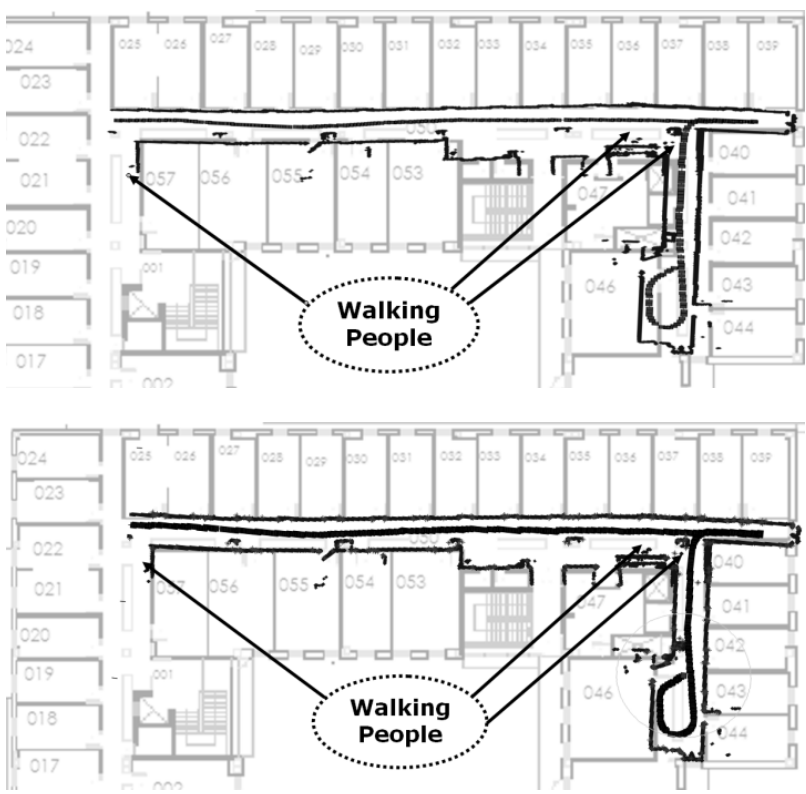

Figure 7. Second experiment: SLAM at the third floor of the PIV building. Top: scan matching based map. Bottom: our estimated map.

Figure 7 shows the results for the second experiment, on top the scan matching based map and on bottom our estimated map. The main goal of the second experiment was to test our approach along long corridors, since is well known that scan matching techniques have a tendency to estimate shorter displacements than expected. This fact can be observed in the first map of Figure 7, where the top-right corner of the office number 053 shows a displacement to the left. However, our approach corrects this error and the rotation error introduced after the $180^{\circ}$ rotation performed by the robot on the top-right part of the map. The dataset was taken in presence of walking people; despite of this fact, it can be observed that our approach shows that the robot path and the environment representation are consistent with CAD map of this floor.

In the same way as before, Figure 8 shows a zoomed region of the third floor map in order to visualize the consistency in the vertical line positions with respect to the laser scan matching. Observing Figure 8, it can be noted groups of vertical lines put together, these lines correspond to those detected in the frame doors. One of the improvements we are working out is using this information and the LRF trace in order to obtain the 3D planes corresponding to the scene, instead of obtaining them only from the catadioptric image analysis as the approach proposed by [19].

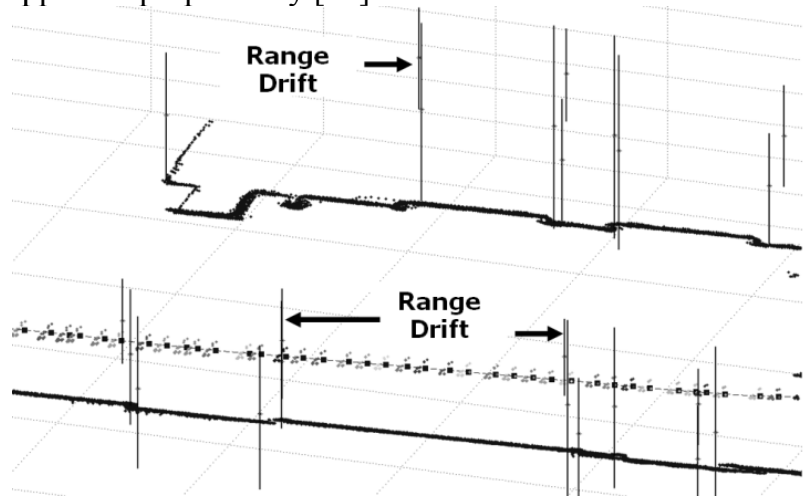

Figure 8. Detail of Figure 7 with 3D vertical lines.

One of the drawbacks of our approach is dealing with scene vertical lines which are very close each other. In this situation, the laser trace projected onto the omnidirectional image is not accurate enough to obtain a good range estimation of the vertical line. The arrows in Figure 6 and Figure 8 show some specific cases. A solution with which we are working is using bilinear interpolation on the image plane.

To verify the filter performance is important to check if the innovations along the robot path fall within the $1-\sigma$ boundaries. Figure 9 shows the range, azimuth and elevation innovations in solid lines, the $1-\sigma$ boundaries and the innovation standard deviation in dotted lines for the experiment one. It can be noted that the innovations are completely into the $1-\sigma$ boundaries, which shows that our particle filter is well matched. 

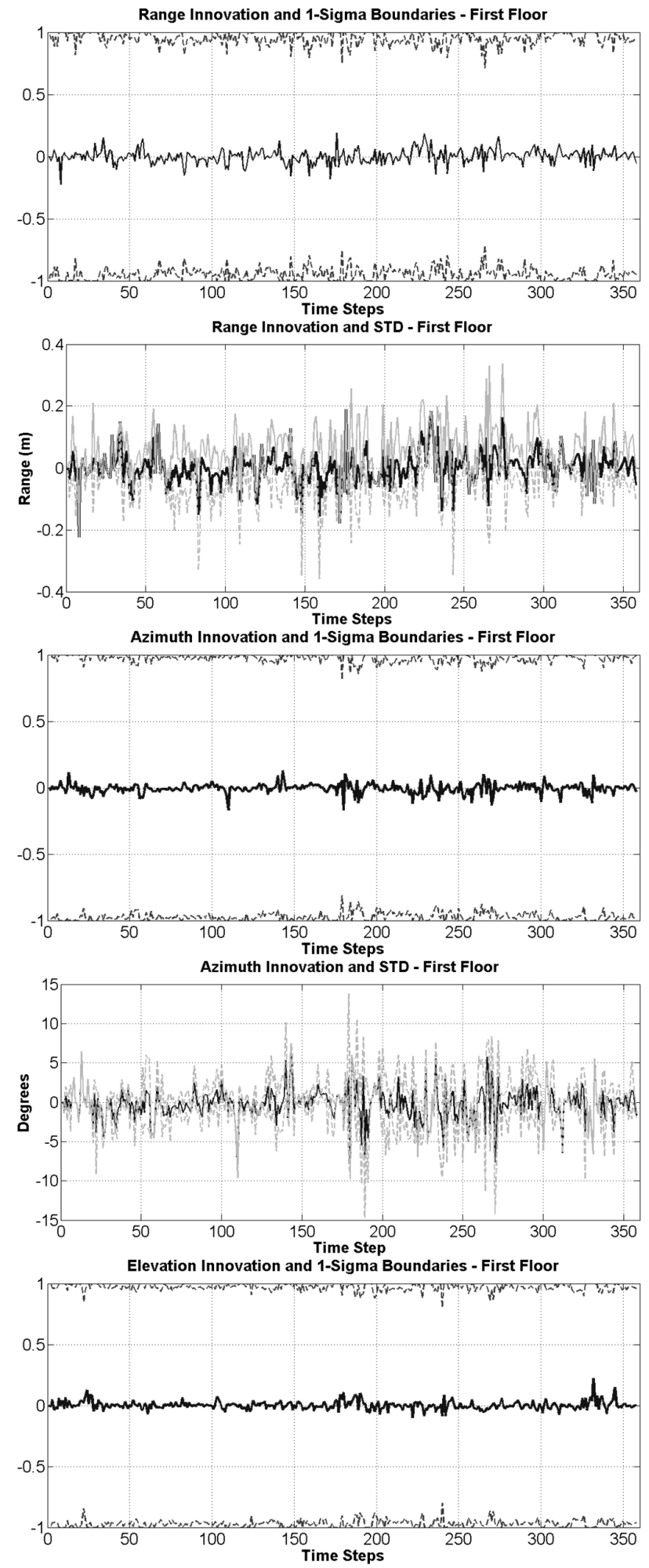

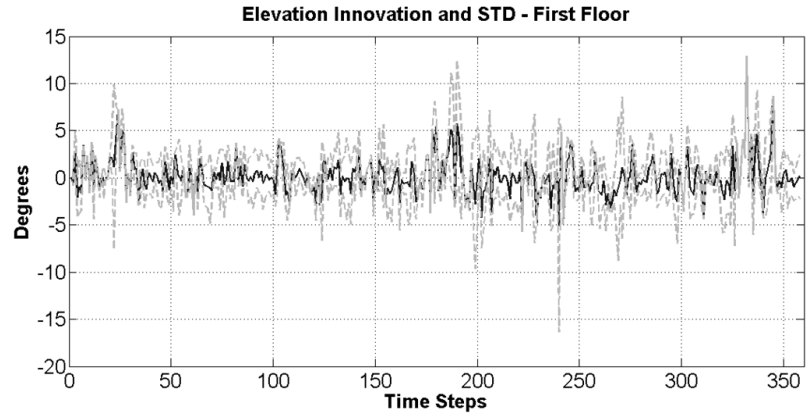

Figure 9. Range, azimuth and elevation innovations with $1-\sigma$ and standard deviation boundaries of experiment one.

\section{Conclusions}

We have presented a range-augmented omnidirectional vision sensor to solve the SLAM problem using a FastSLAM approach. The sensor model presented is based on the extrinsic calibration of a LRF and an omnidirectional camera. Using this calibration, 3D vertical lines were extracted and considered as observations in our implementation of the FastSLAM algorithm. Our approach provides metric information and appearancebased environmental description using one omnidirectional image and the corresponding LRF trace. Using our approach, two problems are solved: the scale factor and the data association disambiguation in dynamic environments.

Observing the results achieved alternative research directions can be considered in order to improve the approach proposed: exploiting other landmark attributes which could be useful for data association; taking advantage of the metric relationship between the 3D vertical edges and the LRF trace in order to extract the local 3D planes; and improving the loop closure detection using the method proposed in [21], instead of relying it only on the data association implemented in this work.

\section{Acknowledgements}

This work has been partially funded by the Commission of Science and Technology of Spain (CICYT) through the coordinated project DPI-2007-66796-C03-02, the LASPAU-COLCIENCIAS grant 136-2008, the University of Valle contract 644-19-04-95, and the consolidated research group's grant SGR2005-01008.

\section{References}

[1] Durrant-Whyte, H.; Bailey, T. Simultaneous Localization and Mapping (SLAM): Part I The Essential Algorithms. Robotics and Automation Magazine 13: 99-110, 2006.

[2] Bailey, T., Durrant-Whyte, H.F., Simultaneous Localisation and Mapping (SLAM): Part II - State of the Art. Robotics and Automation Magazine, 13:108-117, 2006. 
[3] J.Nieto, T.Bailey and E.Nebot, Recursive scan-matching SLAM, Robotics and Autonomous Systems, 55: 39-49, 2007.

[4] P.J. Besl and N.D. Mackay, A method for registration of 3D shapes, IEEE Transactions on Pattern Analysis and Machine Intelligence, 14: 239-256, 1992.

[5] Silveira, G.; Malis, E.; Rives, P., An Efficient Direct Approach to Visual SLAM, Robotics, IEEE Transactions on , 24(5): 969-979, 2008.

[6] Mark Cummins and Paul Newman, Highly Scalable Appearance-Only SLAM - FAB-MAP 2.0, In Robotics Science and Systems (RSS). Seattle, USA. 1-8. 2009.

[7] Ranganathan, A. \& Dellaert, F., Online Probabilistic Topological Mapping,. The International Journal of Robotics Research, 30: 755-771. 2011.

[8] Wongphati, M.; Niparnan, N.; Sudsang, A., Bearing only FastSLAM using vertical line information from an omnidirectional camera, Robotics and Biomimetics, IEEE Int. Conf. on , 1188-1193, 2009.

[9] Scaramuzza, D. Omnidirectional Vision: from Calibration to Robot Motion Estimation, PhD thesis, ETH Zurich, 2008.

[10] Gallegos, G.; Meilland, M.; Rives, P.; Comport, A.I., Appearance-based SLAM relying on a hybrid laser/omnidirectional sensor, Intl. Robots and Systems, IEEE/RSJ Int. conf. on , 3005-3010. 2010.

[11] C. Mei. Laser-Augmented Omnidirectional Vision for 3D Localisation and Mapping. PhD thesis, INRIA Sophia Antipolis, France, 2007.

[12] Bacca, E.B.; Mouaddib, E.; Cufi, X.; "Embedding range information in omnidirectional images through laser range finder," Intell. Robots and Syst. IROS, IEEE/RSJ Int. Conf. on , 2053-2058, 18-22. 2010.

[13] Neira, J.; Tardos, J.D.; Data association in stochastic mapping using the joint compatibility test, Robotics and Automation, IEEE Transactions on , 17(6): 890-897, 2001.

[14] Montemerlo, M.; Thrun, S.; Simultaneous localization and mapping with unknown data association using FastSLAM, Robotics and Automation, 2003. Proceedings. ICRA ' 03. IEEE International Conference on , 2: 1985- 1991, 2003.

[15] S. Thrun, W. Burgard, and D. Fox. Probabilistic Robotics. MIT Press, Cambridge, MA, 2005.

[16] C.Geyer and K.Daniilidis, A Unifying Theory for Central Panoramic Systems and Practical Applications, in European Conference on Computer Vision, 445-461. 2000.

[17] Mei, C.; Rives, P.; Calibration between a central catadioptric camera and a laser range finder for robotic applications, Robotics and Automation, ICRA. Proceedings IEEE International Conference on , 532-537, 2006.

[18] C. Mei, Calibration Toolbox for omnidirectional cameras. http://www.robots.ox.ac.uk/ cmei/Toolbox.html. 2011.

[19] Bazin, J.-C.; Inso Kweon; Demonceaux, C.; Vasseur, P.; Rectangle Extraction in Catadioptric Images," Computer Vision, 2007. ICCV 2007. IEEE 11th International Conference on , 14-21, 2007.

[20] Hokuyo Automatic Co. Ltd, http://www.hokuyo-aut.jp, 2011.

[21] B. Bacca, J. Salvi, X. Cufí, Appearance-based mapping and localization for mobile robots using a feature stability histogram, Robotics and Autonomous Systems, 59(10): 840-857, 2011. 